\title{
Purple wheat alleviates dyslipidaemia in rat model
}

\author{
Suque $\mathrm{LAN}^{1+}$ (D), Yaning MENG ${ }^{2 \dagger}$, Mian Wang ${ }^{3}$, Jian YANG ${ }^{4}$, Guangwei $\mathrm{LI}^{1}$, Rongfei MOU ${ }^{3}$, Yelun ZHANG ${ }^{1^{*}}$, \\ Xingpu $\mathrm{LI}^{*}$, Feng $\mathrm{CHEN}^{3}$, Ranran $\mathrm{BI}^{3}$, Yanyan $\mathrm{ZHAO}^{1}$
}

\begin{abstract}
To study the exact effect of purple wheat (PW) on dyslipidaemia in rats so as to lay the foundation of a dietary therapy for hyperlipidaemia. A total of 42 rats were randomly divided into two groups: normal control group (NC) consisting of 12 rats were fed with standard diet in the whole testing process, the other group rats were fed with high-fat diet to induce dyslipidaemia. The dyslipidaemic rats were averagely divided into three groups: dyslipidaemia control group (DC) with $60 \%$ common wheat flour, PW1 group with diet containing 60\% purple wheat Jizimail flour, PW3 group with 60\% purple wheat Jizimai3 flour. Six weeks later, the weight-gain-ratio of the rats in PW1 and PW3 groups were lower as compared to the rats in DC and NC groups. Further, we found that the levels of triglyceride, total cholesterol and low-density lipoprotein in the PW1 and PW3 groups were significantly reduced to almost that of normal levels. Finally, the results of hematoxylin and eosin staining showed that intaking of Jizimail and Jizimai3 could repair hepatocyte steatosis and kidney injury due to dyslipidaemia. Purple wheat diet therapy reduced lipid metabolism disorders and the liver tissue and renal injury in hyperlipidaemic rats.
\end{abstract}

Keywords: purple wheat; hyperlipidaemia; body weight; liver; kidney.

Practical Application: Purple wheat plays an important role in alleviating lipid metabolism disorders and reducing hepatocyte steatosis and renal injury.

\section{Introduction}

Cardiovascular and cerebrovascular diseases are a major threat to human health. Hyperlipidaemia is a potential and independent risk factor (De Moura et al., 2009; Hong et al., 2012; Navar-Boggan et al., 2015) for various cardiovascular and cerebrovascular diseases including atherosclerosis and coronary heart disease. In order to decrease the morbidity and mortality rates of cardiovascular and cerebrovascular diseases, it is important to prevent hyperlipidaemia (Bolton-Smith et al., 1991), for which dietary therapy and good lifestyle are important (Rosenthal, 2000; Jeon et al., 2001; Jenkins et al., 2003; Jacobson et al., 2015; Yu et al., 2015; Gao et al., 2017). Therefore, the prevention of cardiovascular and cerebrovascular diseases by consumption of foods with lipid-lowering activity has become a hot topic in the field of nutrition science. There are many reports (Vahouny, 1982; Hassona, 1993; Kahlon \& Chow, 1997; Lecumberri et al., 2007) about the effect of dietary fibre on blood lipid. A study (Zhang et al., 2016) concluded that dietary fibre composed of wheat, corn, grains and beans can significantly reduce the levels of triglyceride (TG), total cholesterol (TC) and low-density lipoprotein (LDL) in hyperlipidaemic rats. Generally, dietary fibre from a mixture of grains and beans is more effective than a single dietary fibre of wheat or corn. Lundin et al. (2004) that whole wheat bread can significantly reduce the TC and TG levels in patients with colitis, Leinonen et al. (2000) demonstrated that whole purple wheat bread can effectively reduce the serum levels of TC and LDL in men. The most recent report (Liu, 2017) that black whole wheat flour can significantly reduce serum levels of TC, TG and LDL, and increase serum levels of the high-density lipoprotein (HDL) in hyperlipidaemic rats. These findings suggest that purple wheat can alleviate lipid metabolism disorders.

Purple wheat is nutritionally valuable (Li et al., 2002; Liu et al., 2002; Li et al., 2003; Jaafar et al., 2013; Lan et al., 2013), and it contains many microelements, amino acids, proteins, natural melanin and anthocyanins. Our previous report (Lan et al., 2013) demonstrated that the bread made from Jiziheiixoamail (also named Jizi439), a new purple wheat, could reduce the levels of postprandial blood glucose in diabetic patients, and different purple wheat varieties had different effects on the postprandial blood glucose levels. However, the role of purple wheat in reducing fat and the differences in lipid-lowering effects among different varieties of purple wheat remains unknown. To address these problems, we explored the effects of different purple wheat varieties on weight gain and lipid metabolism in hyperlipidaemic rats. We believe that this study will provide a theoretical basis for the prevention and treatment of diseases caused by a high-fat diet.

Received: 27 Jan., 2021

Accepted: $26 \mathrm{Feb}, 2021$

${ }^{1}$ Institute of Cereal and Oil Crops - ICOC, Hebei Academy of Agriculture and Forestry Sciences - HAAFS, Hebei Province, P. R. China

${ }^{2}$ Institute of Cash Crops - ICC, Hebei Academy of Agriculture and Forestry Sciences - HAAFS, Hebei Province, P. R. China.

${ }^{3}$ The Second Hospital of Hebei Medical University - SHHMU, Hebei Province, P. R. China

${ }^{4}$ Shijiazhuang Agricultural and Rural Bureau-SARB, Shijiazhuang, Hebei Province, P. R. China.

*Corresponding author: zhang-ye-lun@126.com, lixingpu@126.com

${ }^{\dagger}$ Equal contributors: Yaning Meng and Suque Lan led the implementation of the majority project, conducted majority of the data collection and analysis, and drafting the manuscript. Yaning Meng and Suque Lan contributed to the study equally. 


\section{Materials and methods}

\subsection{Sample}

Two varieties of purple wheat (Jizimail, also mamed Jizi439, and Jizimai3) were selected for the study, with $15 \%$ of the bran removed. Flour of the two varieties of purple wheat was obtained from the Institute of Cereal and Oil Crops, the Hebei Academy of Agricultural and Forestry Sciences (Shijiazhuang, China). Common wheat flour was purchased from the supermarket. The feed used in the experiment was produced by the experimental animal center of the Hebei Medical University (Shijiazhuang, China).

High-density lipoprotein cholesterol detection Kit (k075) and low-density lipoprotein cholesterol detection Kit (k076) were purchased from Changchun Huili Biotechnology Co., Ltd. (Changchun, China). A triglyceride detection kit (auz3592) and total cholesterol detection kit (auz3625) were purchased from Beckman Kurt Experimental System Co., Ltd. (Suzhou, China). Cholesterol and sodium cholate were purchased from Henan yipinshihua Co., Ltd. (Zhengzhou, China); yolk powder was purchased from Beijing Kaiyuan Feed Co., Ltd. (Beijing, China); and lard was obtained from Linyi Xincheng Jinluo Meat Products Group Co., Ltd. (Linyi, China).

\subsection{Experimental animals}

Forty-two male SPF (Specific Pathogen Free) grade SD (Sprague-Dawley), weighing 180-210 g, provided by Liaoning Changsheng Biotechnology Co., Ltd. (Benxi, China), license No.: scxk (Liao) 2015-0001, were used for the study. Forty-two rats were raised in separate cages in a controlled environment $\left(22{ }^{\circ} \mathrm{C} \pm 1{ }^{\circ} \mathrm{C}, 12\right.$-h light/12-h dark cycle). Rats were fed a common diet ad libitum and provided with filtered tap water for 7 days to acclimatize to their new environment.

\subsection{Diets and experimental design}

The study followed the national guidelines for care and use of animals, and all experimental procedures were approved by the Animal Ethics Committee at the second hospital of the Hebei Medical University (Shijiazhuang, China; Approval Number: 30309-02-67). Forty-two rats were randomly divided into two groups. The normal control (NC) group consisted of 12 rats which were fed with a standard diet in the whole testing process. The model group consisted of 30 rats which were fed with a high-fat diet, after 4 weeks of feeding, blood was collected from the tail vein and serum levels of TC, TG, LDL and HDL were determined. Serum levels of TC, TG and LDL were significantly higher in the model group than in the control group ( $p<0.05$ ). This indicated (Gao et al., 2017) that dyslipidaemia models were successfully established.

The thirty hyperlipidaemic rats were randomly divided into three groups, 10 rats in each group. The dyslipidaemia control (DC) group was fed with a diet containing $60 \%$ common wheat flour; the purple wheat 1 (PW1) group was fed with a diet containing $60 \%$ purple wheat Jizimail flour; and the purple wheat 3 (PW3) group was fed with a diet containing $60 \%$ purple wheat Jizimai3 flour. The composition of the experimental diets is presented in Table 1, and nutrition composition of different diet has been shown in Table 2. The rats were fed

Table 1. Composition of experimental diets ( $\mathrm{g} / \mathrm{kg}$ of mixture).

\begin{tabular}{|c|c|c|c|c|c|}
\hline \multirow{2}{*}{ Ingredients } & high-fat & standard & DC & PW1 & PW3 \\
\hline & diet & diet & group & group & group \\
\hline purple wheat flour & - & - & - & 600 & 600 \\
\hline Common wheat flour & 197 & 250 & 600 & - & - \\
\hline Corn flour & 213 & 270 & - & - & - \\
\hline Soybean meal & 166 & 210 & 130 & 130 & 130 \\
\hline Wheat bran & 118 & 150 & 150 & 150 & 150 \\
\hline Fish meal & 63 & 80 & 80 & 80 & 80 \\
\hline vitamins & 10 & 15 & 15 & 15 & 15 \\
\hline Fish liver oil & 12 & 15 & 15 & 15 & 15 \\
\hline Slat & 10 & 10 & 10 & 10 & 10 \\
\hline cholesterol & 10 & - & - & - & - \\
\hline sodium cholate & 2 & - & - & - & - \\
\hline lard & 100 & - & - & - & - \\
\hline yolk powder & 100 & - & - & - & - \\
\hline
\end{tabular}

Table 2. Nutrition compositions of the diets fed to rat.

\begin{tabular}{|c|c|c|c|c|c|}
\hline \multirow{2}{*}{ Nutrient compositions } & high-fat & standard & $\mathrm{DC}$ & PW1 & PW3 \\
\hline & diet & diet & group & group & group \\
\hline Total calorie(kcal) & 399.25 & 311.45 & 301.20 & 304.70 & 307.60 \\
\hline Total protein $(\mathrm{g} / 100 \mathrm{~g})$ & 14.77 & 16.80 & 16.30 & 17.60 & 17.80 \\
\hline Total fat $(\mathrm{g} / 100 \mathrm{~g})$ & 16.19 & 5.70 & 4.00 & 4.00 & 4.00 \\
\hline Total carbohydrate $(\mathrm{g} / 100 \mathrm{~g})$ & 36.55 & 45.60 & 51.20 & 49.30 & 51.60 \\
\hline Total fiber $(\mathrm{g} / 100 \mathrm{~g})$ & 8.86 & 10.80 & 9.40 & 9.70 & 7.20 \\
\hline Total vitamins (g/100g) & 1.00 & 1.50 & 1.50 & 1.50 & 1.50 \\
\hline Total minerals (mg) & 1816.11 & 2281.70 & 2056.56 & 2359.99 & 2627.31 \\
\hline
\end{tabular}


regularly and quantitatively every day for six weeks and their daily activities were observed. The bedding and drinking water were changed regularly and the weight of the rats was monitored once a week. At the end of the experiment, all rats were fasted overnight and euthanized using $4 \%$ fluothane in oxygen anaesthesia in an airtight container. Before euthanasia, blood was collected from the inner canthus vein at $3000 \mathrm{rmp}$ for $15 \mathrm{~min}$ at $4{ }^{\circ} \mathrm{C}$. The resulting supernatant was stored at $-80^{\circ} \mathrm{C}$ for biochemical analysis. Liver and kidney were excised, weighed, and stored at $-80^{\circ} \mathrm{C}$. Flowchart of the experiment is presented in Figure 1.

\subsection{Blood lipid estimation}

The serum levels of TC, TG, HDL and LDL were measured according to the kit manufacturer's instructions.

\subsection{Histopathological examination}

For haematoxylin eosin (HE) staining, the liver and the kidneys of the rats were washed in cold $0.9 \%$ normal saline, and the weight of each organ was measured, the liver and the kidneys samples were fixed in $4 \%$ polyformaldehyde and embedded in paraffin. The organs were then sectioned and the sections were stained with haematoxylin and eosin, and observed under $10 \times$ and $40 \times$ magnification of a microscope.

\subsection{Statistical analysis}

Statistical analysis was performed using the Statistical Product and Service software, version 19.0 (SPSS, USA). The data have been expressed as mean \pm SEM. Comparisons between two groups were performed using independent samples t-test. $p<0.05$ was considered statistically significant.

\section{Results and discussion}

\subsection{Effect of purple wheat on the body weight of hyperlipidaemic rats}

The weight of the rats was measured once a week. It was observed that the body weight of rats continuously increased up to six weeks; NC group rats had the highest body weight, followed by DC and PW 1 group rats, and PW3 group rats had the lowest weight. In this study, we observed that the weight-gain-ratio of the PW1 and PW3 group rats was significantly lower than that of the DC group and the NC group rats (Figure 2), which was in agreement with previous reports (Liu, 2017). Furthermore, we found that the weight-gain-ratios of the PW3 group rats were significantly lower than that of the PW1 group rats in the sixth week $(p<0.05)$, Therefore, purple wheat could lead to a reduction of weight gain in hyperlipidaemic rats, and different purple wheat varieties had different effects in reducing the weight gain.

\subsection{Effect of purple wheat on blood lipid levels in hyperlipidaemic rats}

This result shows that the serum levels of TG, TC and LDL in the PW1, PW3 and DC group rats significantly reduced after a dietary intervention as compared with those before a dietary intervention $(p<0.05)$ (Table 3). As shown in Table 3, compared to the NC group, there was no significant difference in serum levels of TG, TC, HDL and LDL between the DC, PW1 and PW3 groups ( $p>0.05)$, reaching serum levels to that of normalcy. Our results were found to be consistent with those of a study conducted by Liu (Liu, 2017). We found serum levels of TC, TG and LDL decreased to varying levels in the PW1 and PW3 groups as compared with the DC group, although there was no significant difference. Liu (Liu, 2017) reported that Serum

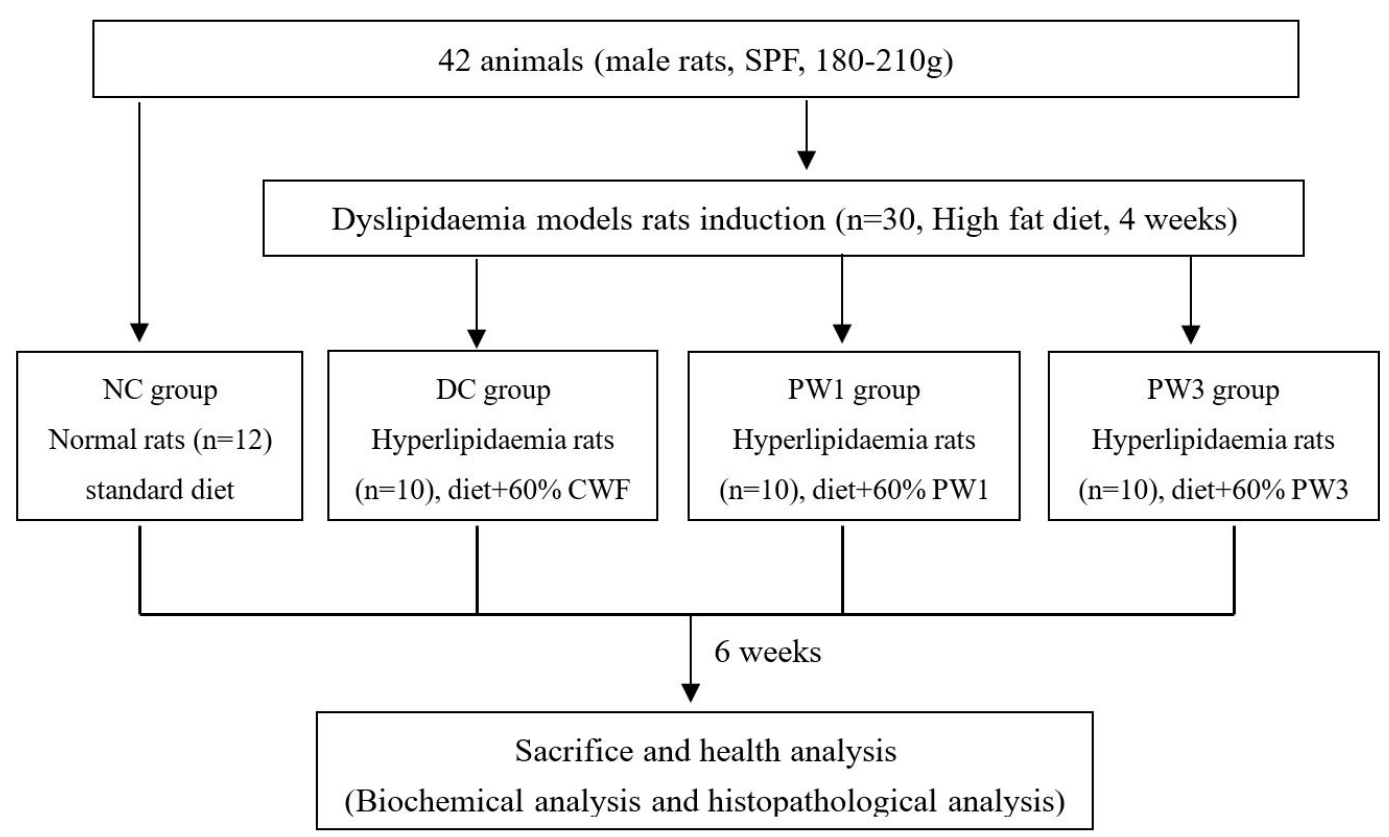

Figure 1. Flowchart of the experiment. $\mathrm{SPF}=$ Specific Pathogen Free; $\mathrm{NC}=$ normal control; $\mathrm{DC}=$ dyslipidaemia control; $\mathrm{CWF}=\mathrm{common}$ wheat flour; PW1 =purple wheat Jizimai1 flour; PW3=purple wheat Jizimai3 flour. 
(A)

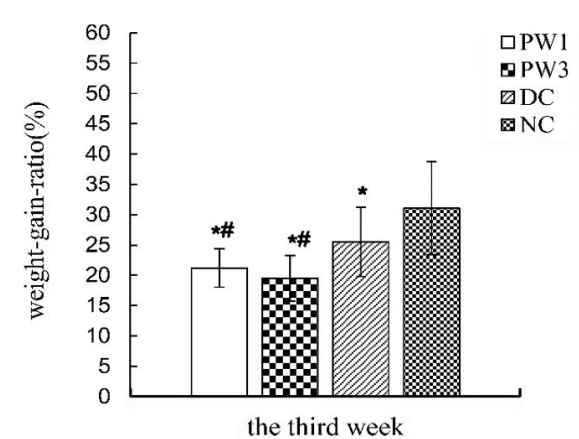

(B)

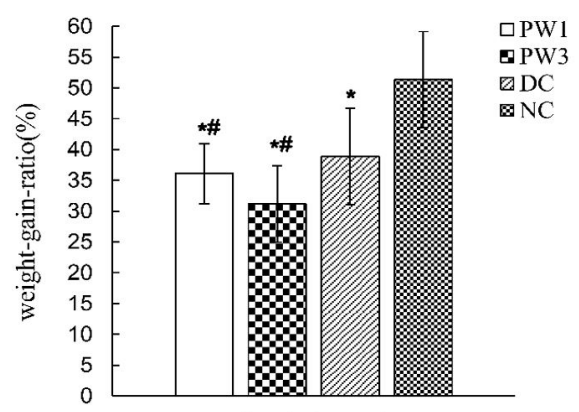

the sixth week

Figure 2. Weight-gain-ratio of rats in each group at different time periods of dietary intervention (A) Weight-gain-ratio of rats at the third week; (B) Weight-gain-ratio of rats at the sixth week. PW1 group: diet containing 60\% purple wheat Jizimai1 flour; PW3 group: diet containing $60 \%$ purple wheat Jizimai3 flour; DC group: dyslipidaemia control group, diet containing 60\% common wheat flour; NC: normal control, standard diet. Data have been expressed as mean \pm S.D. ${ }^{*}$ means $P<0.05$ (vs NC group), \# means $P<0.05$ (vs DC group).

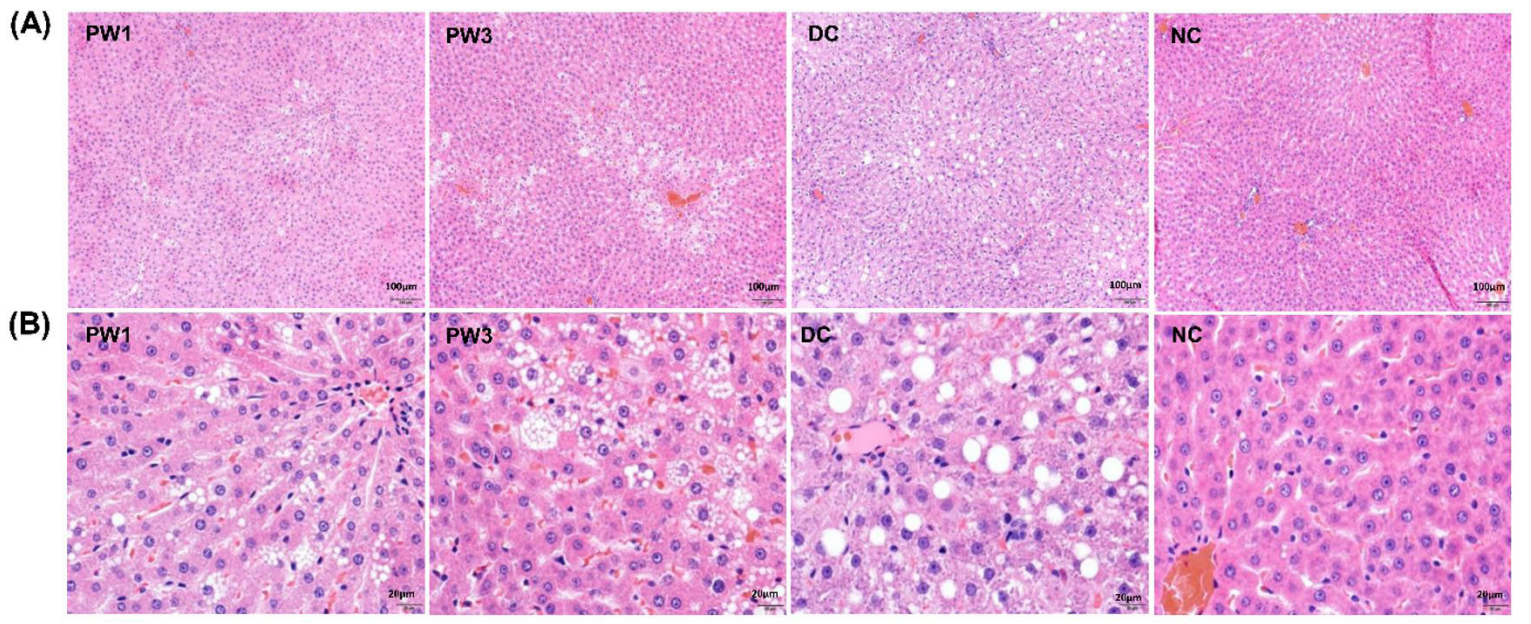

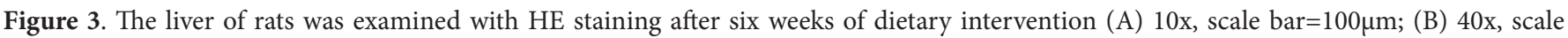
bar $=20 \mu \mathrm{m}$. PW1 group: diet containing 60\% purple wheat Jizimail flour; PW3 group: diet containing 60\% purple wheat Jizimai3 flour; DC group: dyslipidaemia control group, diet containing 60\% common wheat flour; NC: normal control, standard diet.

Table 3. The serum levels of TC、TG、HDL、LDL in different groups.

\begin{tabular}{cccccc}
\hline Time & Group & TG $(\mathrm{mmol} / \mathrm{L})$ & TC $(\mathrm{mmol} / \mathrm{L})$ & HDL $(\mathrm{mmol} / \mathrm{L})$ & LDL $(\mathrm{mmol} / \mathrm{L})$ \\
\hline Before dietary intervention & Model rats & $1.21 \pm 0.46$ & $3.16 \pm 0.42$ & $0.93 \pm 0.16$ & $2.18 \pm 0.29$ \\
dietary intervention for six weeks & PW1 & $0.66 \pm 0.25^{*}$ & $2.14 \pm 0.34^{*}$ & $0.45 \pm 0.06$ & $1.40 \pm 0.30^{*}$ \\
& PW3 & $0.54 \pm 0.20^{*}$ & $2.08 \pm 0.28^{*}$ & $0.41 \pm 0.14$ & $1.43 \pm 0.21^{*}$ \\
& DC & $0.69 \pm 0.31^{*}$ & $2.33 \pm 0.44^{*}$ & $0.57 \pm 0.13$ & $1.44 \pm 0.35^{*}$ \\
Standard diet for six weeks & NC & $0.71 \pm 0.12$ & $2.07 \pm 0.26$ & $0.43 \pm 0.15$ & $1.35 \pm 0.42$ \\
\hline
\end{tabular}

Data are mean \pm SDs, ${ }^{*}$ means $p<0.05$. (compared with Before dietary intervention)

TC and TG significantly decreased, and HDL significantly elevated by the whole purple wheat. The results of this study are inconsistent with those. The reason may be the difference between different varieties of purple wheat, or purple wheat flour without $15 \%$ bran in the present study. It is generally known that dietary fiber is rich in bran, and many studies have reported that the dietary fiber of wheat can reduce the blood lipid level of rats with dyslipidaemia (Bakhsh \& Chughtai, 1984; Zhang et al., 2015). Jing Wang et al. (2011) used wheat bran xylose as a new functional oligosaccharide instead of arabinose, and found that $5 \%$ wheat bran xylose oligosaccharide was beneficial to maintain the weight and lipid homeostasis (Wang et al., 2011). Therefore, whole flour of purple wheat Jizimail and Jizimai3 could be more effective in alleviating dyslipidaemia in rats.

\subsection{Effect of purple wheat on the liver of hyperlipidaemic rats}

The pathological changes in the liver of hyperlipidaemic rats were detected by the HE staining (Figure 3). As compared to the NC group, the DC group showed an unclear liver lobule structure, disordered arrangement of the hepatocyte cords, and disappearance of the cords (Figure 3, DC). Fatty degeneration was observed in almost all the hepatocytes of the DC group. Many 


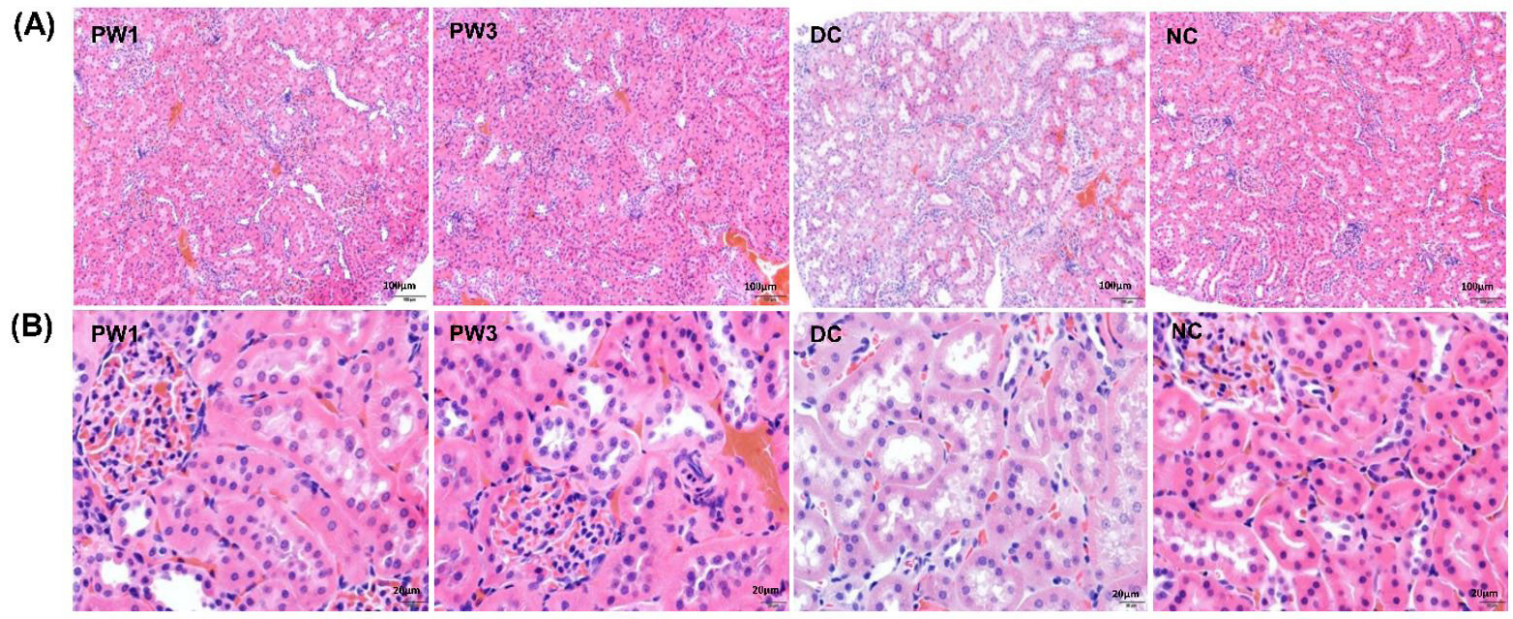

Figure 4. The kidneys of rats were examined with HE staining after six weeks of dietary intervention (A) 10x, scale bar=100 $\mu \mathrm{m}$; (B) 40x, scale bar=20 $\mu \mathrm{m}$. PW1 group: diet containing 60\% purple wheat Jizimail flour; PW3 group: diet containing 60\% purple wheat Jizimai3 flour; DC group: dyslipidaemia control group, diet containing 60\% common wheat flour; NC: normal control, standard diet.

large-area fat vacuoles of different sizes could also be observed. Moreover, the size of hepatocytes was different, and some nuclei were observed to be squeezed and displaced.

The fat denaturation was lighter in PW1 (Figure 3, PW1) and PW3 groups (Figure 3, PW3) as compared to that in the DC group. In PW 1 group, the arrangement of hepatocyte cords was more ordered than that in DC and PW3 groups, and the size of hepatocytes was more uniform than that in PW3 group. However, 30\% of the hepatocytes in PW1 group showed steatosis, and a small number of nuclei were squeezed and displaced. In PW3 group, the arrangement of hepatocyte cords was disordered, fatty degeneration was observed in $60 \%$ of the hepatocytes, and a large number of fat vacuoles were observed in the liver tissue. Moreover, the size of hepatocytes in PW3 group was different, but they were more orderly than those in the DC group, and the phenomenon of a nuclear extrusion and displacement was lighter than that in the DC group. These results showed that purple wheat could attenuate the abnormal liver lipid metabolism and alleviate fatty degeneration in the liver.

\subsection{Effects of purple wheat on the kidneys of hyperlipidaemic rats}

HE staining was used to determine the pathological changes in the kidneys of rats (Figure 4). Compared to the NC group, the DC group showed a loose renal tissue structure (Figure 4, DC), disordered cell arrangement, and evident edema of the glomerular and renal tissue. Moreover, the DC group showed irregular renal tubular epithelial cells, displaced cell nuclei, atrophic cytoplasm in the proximal and distal convoluted tubules, broken cells in the lumen, widened lumen space, and evident renal tissue lesions.

Compared to the DC group, the adverse pathological changes in the kidneys of PW1 (Figure 4, PW1) and PW3 groups (Figure 4, PW3) were alleviated. In PW3 group, the renal tissue structure was uniform and orderly, the structure of tubulointerstitial area was clear, and the shape of renal tubuloepithelial cells was regular. Moreover, the size of the cells was consistent, the arrangement was orderly, and the nucleus was in the center of cells. The lumen space was normal and almost no significant pathological changes were seen in the kidneys. In PW1 group, the shape of renal tubular epithelial cells was slightly irregular, whereas all the other characteristics were normal. Therefore, we suggest that purple wheat plays an important role in alleviating the renal injury in hyperlipidaemic rats.

Hyperlipidaemia can lead to liver injury in rats, resulting in an abnormal lipid metabolism and accumulation of lipids in the liver (Calamita \& Portincasa, 2007). The present study showed that after six weeks of dietary intervention, purple wheat reduced the fatty degeneration and alleviated the renal injury. In contrast, the DC group showed fatty degeneration in almost all hepatocytes and evident renal tissue lesions. Therefore, purple wheat flour is more effective than common wheat in protecting the rat liver and kidneys of hyperlipidaemic rats, both the varieties of purple wheat used in this study are rich in nutrients such as minerals, amino acids, proteins, and natural melanin (Li et al., 2002; Liu et al., 2002; Li et al., 2003). A large number of studies have shown that mineral elements can alleviate lipid metabolism disorders in hyperlipidaemic rats (Koo \& Lee, 1988; Zaporowska \& Wasilewski, 1992; Neggers et al., 2001; Wu et al.,2004; Wu et al., 2006; Huster et al., 2007), proper supplementation of zinc, selenium, copper, vanadium and magnesium can alleviate metabolic disorders in rats. Other studies have shown that anthocyanin can regulate lipid metabolism in hyperlipidaemic rats (Xia et al., 2006; Yang et al. 2011). This may be one of the reasons why purple wheat flour has a good lipid-lowering effect as compared to that by the common wheat flour. However, the mechanism by which purple wheat alleviates dyslipidaemia in rats remains unclear and needs further research.

\section{Conclusion}

(1) The weight-gain ratio of the PW1 and PW3 group rats decreased significantly after six weeks of dietary intervention, indicating that purple wheat could regulate the body weight of hyperlipidaemic rats, Jizimai3 being better than Jizimail in 
reducing weight gain. (2) After six weeks of dietary intervention, the levels of serum TG, TC and LDL in hyperlipidaemic rats significantly reduced, suggesting that purple wheat could regulate the blood lipid levels of rats with abnormal lipid metabolism. (3) Moreover, purple wheat diet therapy reduced fatty degeneration of the liver tissue and alleviated lipid metabolism disorders and renal injury in hyperlipidaemic rats. Thus, purple wheat is more effective than the common wheat in alleviating liver and kidney injury in hyperlipidaemic rats.

\section{Acknowledgements}

This research was financially supported by HAAFS Agriculture Science and Technology Innovation Project (2019-2-5-2). The study was also supported by the project of ministry of science and technology of China (2013bad01b02-11), key R \& D project of ministry of science and technology of China (2016yfd0100102-5), HAAFS Agriculture Science and Technology Innovation Project (2019-4-8-3) and Key R \& D Plan of Hebei Province (19226361D).

\section{References}

Bakhsh, R., \& Chughtai, M. I. D. (1984). Effect of wheat flour, Bengal gram flour and corn flour on lipid metabolism in rats. Journal of Nutritional Science and Vitaminology, 30(3), 297-301. http://dx.doi. org/10.3177/jnsv.30.297. PMid:6491775.

Bolton-Smith, C., Woodward M., Smith, W.C.S., Tunstall-Pedoe H. (1991). Dietary and nondietary predictors of serum total and HDLcholesterol in men and women: results from The Scottish Heart Health Study. International Journal of Epidemiology, 20, 95-104. https://doi.org/10.1093/ije/20.1.95.

Calamita, G., \& Portincasa, P. (2007). Present and future therapeutic strategies in non-alcoholic fatty liver disease. Expert Opinion on Therapeutic Targets, 11, 1231-1249. https://doi.org/10.1517/14728222.11.9.1231.

De Moura, F. F., Lewis, K. D., \& Falk, M. (2009). Applying the FDA Definition of Whole Grains to the Evidence for Cardiovascular Disease Health Claims. The Journal of Nutrition, 139(11), 2220S-2226S. http:// dx.doi.org/10.3945/jn.109.112383. PMid:19776180.

Gao, H., Cheng, W., Xu, Q., \& Shao, L. (2017). Dietary Restriction reduces blood lipids and ameliorates liver function of mice with hyperlipidemia. Journal of Huazhong University of Science and Technology, 37(1), 79-86. http://dx.doi.org/10.1007/s11596-0171698-8. PMid:28224418.

Hassona, H. (1993). High fibre bread containing brewer's spent grains and its effect on lipid metabolism in rats. Molecular Nutrition \& Food Research, 37, 576-582. https://doi.org/10.1002/food.19930370609.

Hong, Y., Deng, C., Zhang, J., Zhu, J., \& Li, Q. (2012). Neuroprotective effect of granulocyte colony-stimulating factor in a focal cerebral ischemic rat model with hyperlipidemia. Journal of Huazhong University of Science and Technology. Medical Sciences, 32(6), 872878. http://dx.doi.org/10.1007/s11596-012-1050-2. PMid:23271289.

Huster, D., Purnat, T. D., Burkhead, J. L., Ralle, M., Fiehn, O., Stuckert, F., Olson, N. E., Teupser, D., \& Lutsenko, S. (2007). High copper selectively alters lipid metabolism and cell cycle machinery in the mouse model of Wilson disease. The Journal of Biological Chemistry, 282(11), 8343-8355. http://dx.doi.org/10.1074/jbc.M607496200. PMid:17205981.

Jaafar, S. N. S., Baron, J., Siebenhandl-Ehn, S., Rosenau, T., Böhmdorfer, S., \& Grausgruber, H. (2013). Increased anthocyanin content in purple pericarp $\times$ blue aleurone wheat crosses. Plant Breeding, 132(6), 546-552. http://dx.doi.org/10.1111/pbr.12090.

Jacobson, T. A., Maki, K. C., Orringer, C. E., Jones, P. H., Kris-Etherton, P., Sikand, G., et al. (2015). National lipid association recommendations for patient-centered management of Dyslipidemia: Part 2. Journal of Clinical Lipidology, 9(6), S1-S112. http://doi.org/10.1016/j. jacl.2015.09.002.

Jenkins, D. J. A., Kendall, C. W. C., Marchie, A., Faulkner, D. A., Wong, J. M. W., \& Souza, R. D. (2003). Effects of a dietary portfolio of cholesterol-lowering foods VS lovastatin on serum lipids and C-reactive protein. Journal of the American Medical Association, 290(4), 502510. http://dx.doi.org/10.1001/jama.290.4.502. PMid:12876093.

Jeon, T. L., Lim, B. O., Yu, B. P., Lim, Y., Jeon, E. J., \& Park, D. K. (2001). Effect of dietary restriction on age-related increase of liver susceptibility to peroxidation in rats. Lipids, 36(6), 589-593. http:// dx.doi.org/10.1007/s11745-001-0761-1. PMid:11485162.

Kahlon, T. S., \& Chow, F. I. (1997). Hypocholesterolemic effects of oat, rice, and barley dietary fibers and fractions. Cereal Foods World, 42, 86-92. http://dx.doi.org/10.1016/S0924-2244(97)80956-9.

Koo, S. I., \& Lee, C. C. (1988). Compositional changes in plasma highdensity lipoprotein particles in marginally zinc-deficient male rats. The American Journal of Clinical Nutrition, 47(1), 120-127. http:// dx.doi.org/10.1093/ajcn/47.1.120. PMid:3337030.

Lan, S., Meng, Y., Li, X., Zhang, Y., Song, G. Y., \& Ma, H. J. (2013). Effect of consumption of micronutrient enriched wheat steamed bread on postprandial plasma glucose in healthy and type 2 diabetic subjects. Nutrition Journal, 12(1), 64. http://dx.doi.org/10.1186/1475-289112-64. PMid:23680007.

Lecumberri, E., Goya, L., Mateos, R., Alía, M., Ramos, S., IzquierdoPulido, M., \& Bravo, L. (2007). diet rich in dietary fiber from cocoa improves lipid profile and reduces malondialdehyde in hypercholesterolemic rats. Nutrition, 23(4), 332-341. https://doi. org/10.1016/j.nut.2007.01.013.

Leinonen, K. S., Poutanen, K. S., \& Mykkänen, H. M. (2000). Rye bread decreases serum total and LDL cholesterol in finnish men with moderately elevated serum cholesterol. The Journal of Nutrition, 130(2), 164-170. http://dx.doi.org/10.1093/jn/130.2.164. PMid:10720164.

Li, X., Hou, H., Liu, Y., Lan, S., \& Zhu, Y. (2002). Studies of Grain Nutritional Quality on Wheat with Blue or Purple Kernels. Huabei Nongxuebao, 17(1), 21-24. http://dx.doi.org/10.3321/j.issn:10007091.2002.01.005.

Li, X., Lan, S., \& Liu, Y. (2003). Studies on Pigment and It $₫$ s Related Physio-biochemical Properties of Blue or Purple Grain Wheat. Zuo Wu Xue Bao, 29, 157-158.

Liu, H. (2017). Regulatory function of rye whole wheat flour on lipid metdbolism disorder rats and hyperglycemia mice (Master's dissertation). Shandong University, Jinan, China. Retrieved from https://cdmd. cnki.com.cn/Article/CDMD-10422-1017081305.htm

Liu, Y., Quan, S., Li, X., Lan, S., Liu, Y., \& Li, J. (2002). Protein Content and Acid Composition and Qualities of Different Blue or Purple Grain Wheat. Huabei Nongxuebao, S1, 103-107. http://dx.doi. org/10.3321/j.issn:1000-7091.2002.z1.022.

Lundin, E. A., Zhang, J. X., Lairon, D., Tidehag, P., Aman, P., Adlercreutz, H., \& Hallmans, G. (2004). Effects of meal frequency and high-fibre rye-bread diet on glucose and lipid metabolism and ileal excretion of energy and sterols in ileostomy subjects. European Journal of Clinical Nutrition, 58(10), 1410-1419. http://dx.doi.org/10.1038/ sj.ejcn.1601985. PMid:15100716.

Navar-Boggan, A.M., Peterson, E.D., D’Agostino, R.B., Neely, B., Sniderman, A.D., Pencina, M.J., et al. (2015). Hyperlipidemia in early adulthood 
increases long-term risk of coronary heart disease. Circulation, 131(5), 451-458. http://doi.org/10.1161/CIRCULATIONAHA.114.012477.

Neggers, Y. H., Bindon, J. R., \& Dressler, W. W. (2001). The relationship between zinc and copper status and lipid levels in African-Americans. Biological Trace Element Research, 79(1), 1-13. http://dx.doi. org/10.1385/BTER:79:1:01. PMid:11318232.

Rosenthal, R. L. (2000). Effectiveness of altering serum cholesterol levels without drugs. Proceedings, 13(4), 351-355. http://dx.doi.or g/10.1080/08998280.2000.11927704. PMid:16389340.

Vahouny, G. V. (1982). Dietary fiber, lipid metabolism, and atherosclerosis. Federation Proceedings, 41(11), 2801-2806. http://dx.doi.org/10.1016. S0065-3233(08)60471-4. PMid:6288480.

Wang, J., Cao, Y., Wang, C., \& Sun, B. (2011). Wheat bran xylooligosaccharides improve blood lipid metabolism and antioxidant status in rats fed a high-fat diet. Carbohydrate Polymers, 86(3), 1192-1197. http:// dx.doi.org/10.1016/j.carbpol.2011.06.014.

Wu, B., Liu, H., \& Liu, X. (2006). Effect on Mineral Metabolism and Lipid Metabolism in Mice Fed with High-fat Food. Trace Elements Science, 13(7), 26-29.

Wu, Y., Sun, Z., Che, S., Chang, H., \& Wang, Y. (2004). Effects of zinc and selenium on the disorders of blood glucose and lipid metabolism and its molecular mechanism in diabetic rats. Wei Sheng Yen Chiu, 33(1), 70-73. PMid:15098483.

Xia, X., Ling, W., Ma, J., Xia, M., Hou, M., Wang, Q., Zhu, H., \& Tang, Z. (2006). An anthocyanin-rich extract from black rice enhances atherosclerotic plaque stabilization in apolipoprotein E-deficient mice. The Journal of Nutrition, 136, 2220-2225. https://doi.org/10.1093/ jn/136.8.2220.

Yang, Y., Andrews, M. C., Hu, Y., Wang, D., Qin, Y., Zhu, Y., Ni, H., \& Ling, W. (2011). Anthocyanin extract from black rice significantly ameliorates platelet hyperactivity and hypertriglyce-ridemia in dyslipidemic rats induced by high fat diets. Journal of Agricultural and Food Chemistry, 59(12), 6759-6764. http://dx.doi.org/10.1021/ jf201079h. PMid:21568342.

Yu, Z., Wang, R., Fok, W. C., Coles, A., Salmon, A. B., \& Pérez, V. I. (2015). Rapamycin and dietary restriction induce metabolically distinctive changes in mouse liver. The Journals of Gerontology. Series A, Biological Sciences and Medical Sciences, 70(4), 410-420. http:// dx.doi.org/10.1093/gerona/glu053. PMid:24755936.

Zaporowska, H., \& Wasilewski, W. (1992). Combined effect of vanadium and zinc on certain selected haematological indices in rats. Comparative Biochemistry and Physiology, 103(1), 143-147. http://dx.doi.org/10.1016/0742-8413(92)90243-Z. PMid:1360368.

Zhang, H., Jiang, M. X., Cheng, Y., Ye, B., \& Zhai, C. (2016). Effects of three kinds of dietary fiber on glucolipid metabolism and oxidative stress in lipid metabolism disorder rats. Journal of Southeast University, 35, 83-85.

Zhang, W., Jiao, J., Qin, L., Li, L., Han, S., \& Fu, C. (2015). Effects of cereal dietary fiber on lipid metabolism and lipotoxicity in mice fed with high fat/cholesterol diet. Chinese Journal of Public Health, 31, 1038-1040. http://dx.doi.org/10.11847/zgggws2015-31-08-16. 